3. Berkow SE, Freed LM, Hamosh M, Bitman J, Wood DL, Happ B et al. Lipases and lipids in human milk; effect of freeze- thawing and storage. Pediatr Res. 1984;18: 1257-62.

4. Lavine M, Clark RM. Changing pattern of FFA in breastmilk during storage. J Pediatr Gastroenterol Nutr. 1987;6:769-74.
5. Fidler N, Sauerwald TU, Koletzko B, Demmelmair H. Effects of human milk pasteurization and sterilization on available fat content and fatty acid composition. J Pediatr Gastroenterol Nutr. 1998;132:876-8

6. Thatrimontrichai A, Janjindamai W, Puwanant M. Fat loss in thawed breast milk: Comparison between refrigerator and warm water. Indian Pediatr. 2012;49:877-80 .

\title{
Disease Course in Childhood Steroid Sensitive Nephrotic Syndrome: Is it Changeable?
}

\author{
SHUICHI Ito \\ Division Chief, Division of Nephrology and Rheumatology, National Center for Child Health and Development, \\ 2-10-1 Okura, Setagaya-ku, Tokyo, 157-8535. \\ ito-shu@ncchd.go.jp
}

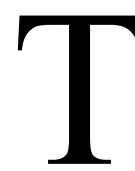

he long-term prognosis of steroid-sensitive nephrotic syndrome is relatively good. However, most patients experience relapses in their clinical course. In this issue, Sinha, et al.

[1] report the prognosis of steroid-sensitive nephrotic syndrome in 1071 children, which is the largest retrospective cohort study to date. They describe that approximately one-half of the patients developed frequent-relapsing nephrotic syndrome (FRNS) or steroid-dependent nephrotic syndrome (SDNS), consistent with the rates in previous reports. FRNS and SDNS are critical issues for children, because of the severe adverse effects of steroid treatment, and psychological issues owing to long hospitalization, frequent hospital visits, and side effects of treatment. Therefore, clarification of risk factors for a frequentrelapsing course and its prevention are quite important. The authors concluded that early onset ( $<4$ years), lack of adequate initial therapy ( $<8$ weeks), and short duration of initial remission lasting $<6$ months are risk factors for a frequent-relapsing course. Among these, the only one in which we can intervene is initial therapy. Although many pediatric nephrologists think that initial steroid treatment at disease onset may influence the future clinical course, the optimal length of initial steroid therapy remains controversial. A standard basic initial steroid therapy at primary onset was proposed by the International Study of Kidney Disease in Children (ISKDC) more than 30 years ago. The proposal was daily steroid therapy $\left(60 \mathrm{mg} / \mathrm{m}^{2} / \mathrm{d}\right)$ for 4 weeks followed by alternate-day treatment $(40 \mathrm{mg} /$ $\mathrm{m}^{2} / 2 \mathrm{~d}$ ) for 4 weeks (original was 3 days of medication followed by 4 days off in a week, but later modified). Thereafter, this protocol was revised in a variety of ways to obtain a better prognosis.
The KDIGO (Kidney Disease: Improving Global Outcomes) guideline recommends that oral prednisone should be administered as a single daily dose starting at $60 \mathrm{mg} / \mathrm{m}^{2} / \mathrm{d}$ or $2 \mathrm{mg} / \mathrm{kg} / \mathrm{d}$ (maximum: $60 \mathrm{mg} / \mathrm{d}$ ) for $4-6$ weeks followed by alternate-day medication at $40 \mathrm{mg} / \mathrm{m}^{2} /$ $2 \mathrm{~d}$ or $1.5 \mathrm{mg} / \mathrm{kg} / 2 \mathrm{~d}$ (maximum: $40 \mathrm{mg} / 2 \mathrm{~d}$ ) with tapering of the dose for 2-5 months [2]. The published US guideline recommends that the initial steroid therapy should be $2 \mathrm{mg} / \mathrm{kg} / \mathrm{d}$ for 6 weeks followed by $1.5 \mathrm{mg} / \mathrm{kg} /$ $2 \mathrm{~d}$ on alternate days for 6 weeks (maximum: $40 \mathrm{mg} / 2 \mathrm{~d}$ ) [3]. In the Cochrane systematic review, it is stated that duration of therapy up to 7 months appeared to be more effective than therapy for 2 months in achieving sustained remission. In Japan, a randomized controlled trial (RCT) comparing daily therapy for 4 weeks followed by alternate-day therapy for 6 months with the standard 2month therapy is ongoing. The results of this RCT will provide some suggestions regarding the optimal mode of initial steroid treatment. The duration of daily steroid therapy, i.e. 4 or 6 weeks, should also be evaluated by an RCT.

Although half of the 1071 patients progressed to FRNS, 185 children had FRNS or SDNS and 42 had late steroid resistance in long-term observation. However, $72 \%$ of FRNS patients were in remission or had infrequent relapses with immunosuppressive agents or low-dose steroid. Therefore, the long-term prognosis is relatively good. However, a certain proportion of patients showed disease transition to the adult form or a difficult clinical course resistant to existing therapy. Such patients were likely to be complicated with severe infection, resulting in high mortality in their study. Recently, rituximab, an anti-CD20 antibody, has become an 
emerging therapy for difficult nephrotic syndrome $[5,6]$. Rituximab allows such patients to discontinue steroid treatment and dramatically reduces the number of relapses. Rituximab can also induce remission in patients with intractable steroid-resistant nephrotic syndrome. Although more clinical experience is needed to establish a safe and effective mode of administration, such new molecular target therapies will improve the clinical course of childhood nephrotic syndrome in the future.

\section{REFERENCES}

1. Sinha A, Hari P, Sharma PK, Gulati A, Kalaivani M, Mantan M, et al. Disease Course in Steroid Sensitive Nephrotic Syndrome. Indian Pediatr. 2012;49:881-7 .

2. Improving Global Outcomes (KDIGO) Glomerulonephritis Work Group. KDIGO Clinical
Practice Guideline for Glomerulonephritis. Kidney Inter. 2012; 2: 139-274.

3. Gipson DS, Massengill SF, Yao L, Nagaraj S, Smoyer WE, Mahan JD, et al. Management of childhood onset nephrotic syndrome. Pediatrics. 2009;124: 747-57.

4. Hodson EM, Willis NS, Craig JC. Corticosteroid therapy for nephrotic syndrome in children. Cochrane Database Syst Rev. 2007; 17:CD001533.

5. Gulati A, Sinha A, Jordan SC, Hari P, Dinda AK, Sharma $\mathrm{S}$, et al. Efficacy and safety of treatment with rituximab for difficult steroid-resistant and dependent nephrotic syndrome: multicentric report. Clin J Am Soc Nephrol. 2010;5:2207-12.

6. Ito S, Kamei K, Ogura M, Sato M, Fujimaru T, Ishikawa T, et al. Maintenance therapy with mycophenolate mofetil after rituximab in pediatric patients with steroid-dependent nephrotic syndrome. Pediatr Nephrol. 2011; 26:1823-8.

\title{
Risk Factors for Mortality in Children Hospitalized with Pneumonia
}

\author{
AK PATWARI \\ Department of Pediatrics, Hamdard Institute of Medical Science \& Research, New Delhi. \\ akpatwari@gmail.com
}

$\mathrm{P}$ neumonia continues to be one of the leading causes of mortality among children under five years of age despite effective vaccines and nutritional and environmental interventions. Pneumonia deaths in health facilities might appear as the 'tip of the iceberg' because most of the deaths are taking place even before these children reach a health facility. However, with improvement in referral services, an increasing proportion of pneumonia deaths are likely to happen in hospitals. Therefore, identification of risk factors of death in hospitalized children suffering from pneumonia is critical for optimal utilization of scarce resources for appropriate management and reduction of childhood mortality.

Significant independent predictors of mortality in children with pneumonia reported earlier by several prospective studies relate to host characteristics (age less than 1 year, lack of exclusive breastfeeding), severity of infection (severe roentgenogram changes, leukocytosis, bandemia), worsening of clinical condition, and hypoxemia (inability to feed, grunting, head nodding, respiratory rate $>70 /$ minute, severe chest indrawing, cyanosis), severe malnutrition (weight for age $\mathrm{Z}$ score $<-3$ ) and associated co-morbidities like diarrhea and HIV [1-4].

In this issue, Ramachandran, et al. [5] attempt to review the situation based on retrospective chart review of
4375 children over a period of 3 years. The case fatality rate (CFR) has been reported as $8.2 \%$ which is comparable to earlier published reports [1]. This study has identified need for assisted ventilation as the single independent risk factor for mortality in children with pneumonia diagnosed by radiological, clinical or clinical and radiological criteria. The results of this study have been primarily interpreted as comparison between 3 study groups, based on certain diagnostic criteria. No significant differences have been noted in the CFR among these study groups. Since it was a retrospective review no hypothesis was made for any expected differences between these groups. However, comparison of data from three different diagnostic/identification groups with studies using similar criteria would have been helpful.

Categorization of cases according to severity at the time of admission is critical to identify 'at risk' children for anticipating the clinical course and to be prepared to handle any complications. A study from Delhi [1] has demonstrated this important association of severity of illness with CFR ranging from $0-47 \%$ in children with pneumonia, severe pneumonia, and very severe pneumonia as per WHO classification. In the study by Ramachandran, et al. [5] 47-68\% of the children who died required assisted ventilation which is much higher than reported by others [2]. It is difficult to understand the 